\title{
Transmission System Reconfiguration to Reduce Losses and Cost Ensuring Voltage Security
}

\author{
Veerapandiyan V. ${ }^{1}$, Mary D. ${ }^{2}$ \\ ${ }^{1}$ Department of Electrical and Electronics Engineering, Rajalakshmi Engineering College, Chennai, India \\ ${ }^{2}$ Department of Electrical Engineering, Government College of Technology, Coimbatore, India \\ Email: veerapandiyan.v@rajalakshmi.edu.in,drmary@gct.ac.in
}

Received 11 April 2016; accepted 10 June 2016; published 13 June 2016

Copyright (C) 2016 by authors and Scientific Research Publishing Inc.

This work is licensed under the Creative Commons Attribution International License (CC BY). http://creativecommons.org/licenses/by/4.0/

c) (i) Open Access

\begin{abstract}
The aim of this paper is to reduce the losses, total generation cost by switching of transmission line and to maintain voltage security under $N-1$ contingency conditions. Generation cost is calculated and the priority list is made for switching the line. The problem is solved by ACOPF using Interior Point Method. In order to test the feasibility and effectiveness of the above method, a sample 6-bus system and IEEE 30-bus system have been used. The impact of switching on system parameter includes the generation cost, locational marginal pricing (LMP) and transmission losses, ensuring voltage security of the system.
\end{abstract}

\section{Keywords}

Transmission Switching (TS), AC Optimal Power Flow (ACOPF), $N-1$ Contingency and Interior Point Method

\section{Introduction}

The electrical energy generated in the power station does not match the demand and some amount of power loss occurs both in transmission and distribution network. The total loss in the transmission line is approximately equal to $17 \%$ in developing countries like India, and it is less than $10 \%$ in developed countries. This loss in the system can be reduced by network switching. Network switching provides flexibility to the system operator, and it is also used as a control method for problems including stability studies, line overloading, loss or cost reduction, congestion management and system security.

There are two types of transmission switching [1]: 
- Sectionalizing switches

- Tie switches

These switches are normally closed or normally open. From time to time the network operator changes the state of these switches in order to enhance security. The network switching can be classified into two main categories [1]: 1) opening or closing branches and 2) substation switching.

Since 1980, some research work has been carried out for network reconfiguration. Switching was first introduced in [2], in which switching was used as preventive control action. In [3] switching is used as a congestion management tool in transmission system, where the problem is solved using Newton's method of ACOPF and the results are compared with DCOPF. It is found that, by means of switching, the generation and transmission parameters change; it is also discussed and the results are compared with DCOPF. In [4] DC load flow and line outage distribution factors have been used to determine the line switching.

Geraldo et al. [2006], [5] suggested a method to solve the nonlinear problem using Interior Point Method based on rectangular coordinates without considering switching. In this paper an approach is given to initialize variables, the efficient assembling of Jacobian and Hessian matrices, sparse data structures, solution of linear system, choice and setting of algorithm is discussed.

In this paper, the OPF problem is solved using Interior Point Method (Primal/dual method), considering switching of transmission lines to reduce system losses. The impact of these on system voltage is studied and the congestion relief due to switching also discussed. The optimal transmission switching is selected based on the minimization of objective function. An OPF is a nonlinear optimization problem with both continuous and discrete variables. The aim of OPF is to optimize the objective function [6] by acting on available control, while satisfying network power flow equations, physical and operational constraints are considered. The interior-point method (IPM) becomes important to solve this OPF problem because of the following features [7]: i) ease of handling inequality constraints by logarithmic barrier functions; ii) speed of convergence and iii) a strictly feasible initial point is not required [5].

Section II gives the problem formulation using IPM and the results are discussed in Section III for both 6-bus and 30-bus system. The impacts on system parameter due to switching are discussed in Section IV with priority list made in the Section V. Finally, the conclusions are made in Section VI.

\section{OPF Using Interior Point Method}

The optimal TS is used as a tool to reduce the system losses and generation cost. Here the number of switching actions in the trial is greater than one and then the priority list is made considering the minimum generation cost. First, ACOPF is run without switching the line [3] and later each line is switched to find the candidate line that actually reduces the losses. The problem is formulated as [8] [9]:

\subsection{Objective Functions}

The objective function to be minimized are generation cost and transmission losses by means of transmission switching, they are

$$
\begin{aligned}
& \min \sum_{i=1}^{g}\left(c_{i}+b_{i} P_{g i}+a_{i} P_{g i}^{2}\right) \\
& \min \sum_{i=1}^{n} \sum_{j=1}^{n} G_{i j}\left[\left(e_{i}-e_{j}\right)^{2}+\left(f_{i}-f_{j}\right)^{2}\right]
\end{aligned}
$$

where $a_{i}, b_{i}, c_{i}$ is the cost coefficients describing its cost curve of generator connected at bus $i$.

\subsection{Equality Constraints}

Equality constraints include both active and reactive power balance equations, for $i^{\text {th }}$ bus $(i=1,2, \cdots, n)$, take the form [10]

$$
\begin{gathered}
P_{g i}-P_{d i}-V_{i}^{2} \sum_{j \in N_{i}}\left(G_{s i j}+G_{i j}\right)+\sum_{j \in N_{i}}\left[\left(e_{i} e_{j}+f_{i} f_{j}\right) G_{i j}+\left(f_{i} e_{j}-e_{i} f_{j}\right) B_{i j}\right]=0 \\
Q_{g i}-Q_{d i}+V_{i}^{2}\left[B_{s i}+\sum_{j \in N_{i}}\left(B_{s i j}+B_{i j}\right)\right]-\sum_{j \in N_{i}}\left[\left(e_{i} e_{j}+f_{i} f_{j}\right) B_{i j}+\left(f_{j} e_{i}-e_{j} f_{i}\right) G_{i j}\right]=0
\end{gathered}
$$


$N_{i}$ is the set of buses connected by branches to the bus $i$.

\subsection{Inequality Constraints}

The Inequality constraints for secure operation of the system,

$$
\left(V_{i}^{\min }\right) \leq e_{i}^{2}+f_{i}^{2} \leq\left(V_{i}^{\max }\right), i=1, \cdots, n
$$

To express constraints on branch real and reactive power is expressed as:

$$
\begin{aligned}
P_{g i}^{\min } & \leq P_{g i} \leq P_{g i}^{\max }, i=1,2, \cdots, g \\
Q_{g i}^{\min } & \leq Q_{g i} \leq Q_{g i}^{\max }, i=1,2, \cdots, g
\end{aligned}
$$

\subsection{Obtaining the Optimality Conditions in the Interior-Point Method [5]}

The OPF formulation in the above method is optimizing the objective function (1) \& (2) subjected to the constraints (5)-(7) can be written as general nonlinear programming problem:

$$
\min f(x) \text { [5] }
$$

Subject to:

$$
\begin{aligned}
& g(x)=0 \\
& h(x) \geq 0
\end{aligned}
$$

where $f(x), g(x) \& h(x)$ are assumed to be continuously differentiable twice, $x$ is an m-dimensional vector that encompasses both the control and state variables (real and imaginary part of voltage at all buses), $g$ is a $\mathrm{n}$-dimensional vector of functions and $h$ is a p-dimensional vector of functions.

This method includes four steps to obtain optimal conditions. First the inequality constraints are transformed to equality constraints by adding slack variables and non-negativity conditions on these:

$$
\min f(x)
$$

Subject to:

$$
\begin{aligned}
& g(x)=0 \\
& h(x)-s=0, s \geq 0
\end{aligned}
$$

Here the vectors $x$ and $s=\left[s_{1}, \cdots, s_{p}\right]^{\mathrm{T}}$ are called primal variables.

By adding logarithmic barrier to objective function the inequality constraints can be eliminated, resulting in the equality constraints as below:

$$
\min f(x)-\mu \sum_{i=1}^{p} \ln s_{i}
$$

Subject to:

$$
g(x)=0, h(x)-s=0
$$

$\mu \rightarrow$ barrier parameter (positive scalar), This is gradually decreased to zero as iteration progress. When the $\mu \rightarrow 0$, the solution of $x(\mu)$ converges to a local optimum of the problem.

By defining Lagrangian function the equality constraint is made as unconstrained one,

$$
L_{\mu}(y)=f(x)-\mu \sum_{i=1}^{p} \ln s_{i}-\lambda^{\mathrm{T}} g(x)-\pi^{\mathrm{T}}[h(x)-s]
$$

where the vectors of Lagrange multipliers $\lambda$ and $\pi$ are called dual variables and $y=[s, \pi, \lambda, x]^{\mathrm{T}}$.

\section{Results and Discussion}

\subsection{Sample 6-Bus System}

The feasibility of above method is tested in sample 6-bus test system [11]. System data is given in [12]. In this 
system there are 3 generators along with 11 transmission lines. Also, the system provides 217.4 MW active powers to serve the loads. An ACOPF problem is run by switching the lines; the optimal switching line called candidate line [13] is selected based on the priority list made by total cost of generation.

Total losses and generation cost:

Table 1 shows the results of total generation cost and losses before and after switching of the line. By switching, the candidate lines are found to be $(1-2),(2-3)$. Among these two lines the most preferred line is (1 - 2) than (2 - 3) because it reduces the cost as well as the losses as shown in Figure 1 \& Figure 2 under $(N-1)$ contingency condition.

\subsection{IEEE-30 Bus Test System}

The IEEE-30 bus system is more realistic and a large test system is used here. It consists of 6 generators, 41 transmission lines with total load of 183.4 MW and 126.2 MVAr. The data for this system is available in [14]. Also the system provides 290.3 MW and 93.4 MVAr to serve the load.

Total losses and generation cost:

Figure 3 and Figure 4 shows the variation of real and reactive power losses before and after switching. The result shows the switching of line 25 - 27 and 24 - 25 reduces the real power losses more than the other switching lines [15]. But the losses are reduced due to subsequent switching of lines as shown in Table 2 when compared with normal case i.e. before opening of line. The total cost of generation variation before and after opening of the transmission line is shown in Figure 5. The results show that the total cost reduced after switching of

Table 1. Total cost, losses and iteration convergence.

\begin{tabular}{cccccc}
\hline System state & Candidate line & Convergence time & TC (\$/hr) & P (MW) & Total loss \\
\hline Base case & - & 0.12 & 3143.97 & 6.908 & 21.21 \\
$\mathbf{1}^{\text {st }} \mathbf{T S}$ & $1-2$ & 0.17 & 3129.64 & 6.617 & 20.60 \\
$\mathbf{2}^{\text {nd }} \mathbf{T S}$ & $2-3$ & 0.14 & 3143.17 & 6.82 & 20.90 \\
\hline
\end{tabular}

$\mathrm{TC}(\$ / \mathrm{h})$

- Total $\operatorname{cost}(\$ / \mathrm{hr})$

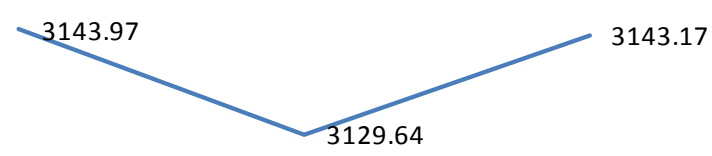

\begin{tabular}{lll}
\hline normal & outage(1-2)
\end{tabular}

Figure 1. Total cost of generation variation.

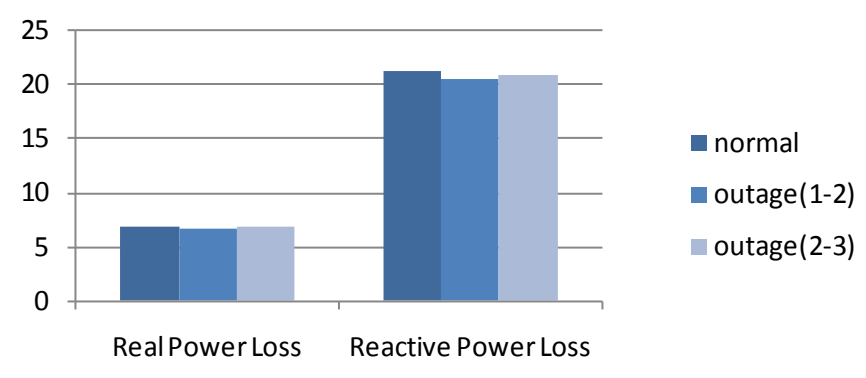

Figure 2. Power losses. 
$\mathrm{P} \operatorname{loss}(\mathrm{MW})$

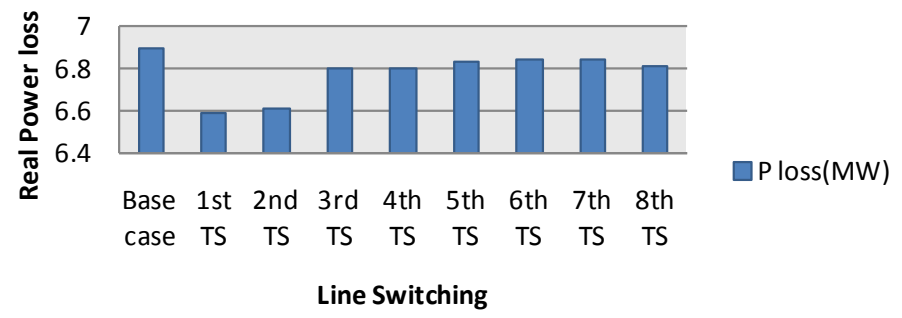

Figure 3. Real power loss.

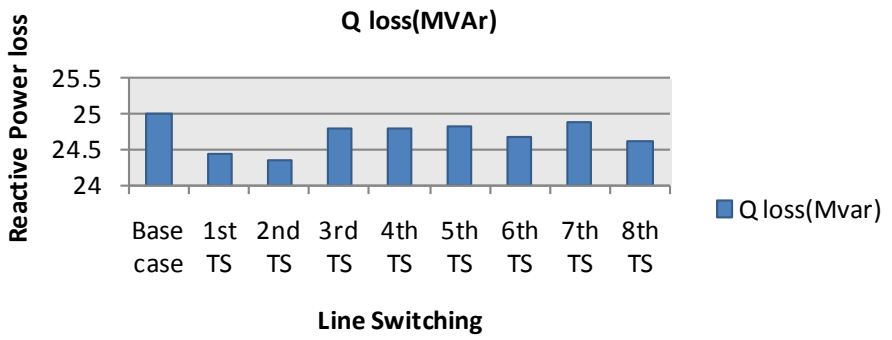

Figure 4. Reactive power loss.

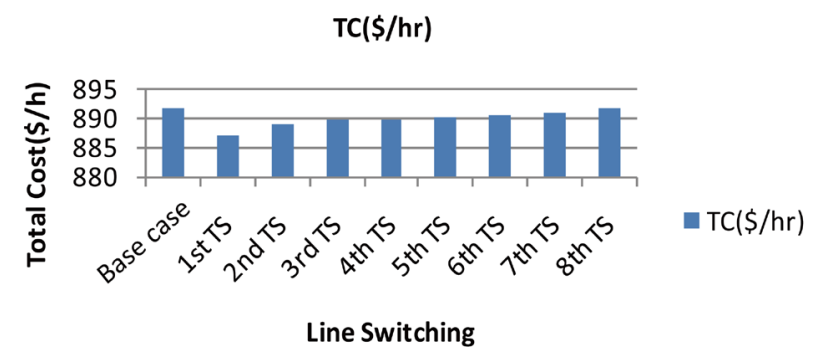

Figure 5. Total cost variation.

Table 2. Total cost, LMP and Loss variation for IEEE-30 bus system.

\begin{tabular}{ccccccccc}
\hline $\begin{array}{c}\text { System state } \\
\text { \& ranking }\end{array}$ & Candidate line & TC $\mathbf{( \$ / \mathbf { h } )}$ & \multicolumn{3}{c}{ LMP $\mathbf{( \$ \mathbf { M W h } )}$} & \multicolumn{3}{c}{ Total loss } \\
\hline Base case & - & 891.67 & 3.94 & 6.71 & 5.325 & 6.89 & 25 \\
$\mathbf{1}^{\text {st }} \mathbf{T S}$ & $25-27$ & 887.18 & 4.04 & 5.05 & 4.545 & 6.594 & 24.45 \\
$\mathbf{2}^{\text {nd }} \mathbf{T S}$ & $24-25$ & 888.71 & 4 & 5.35 & 4.675 & 6.615 & 24.36 \\
$\mathbf{3}^{\text {rd }} \mathbf{T S}$ & $6-9$ & 889.71 & 3.98 & 5.95 & 4.965 & 6.796 & 24.8 \\
$\mathbf{4}^{\text {th }} \mathbf{T S}$ & $9-10$ & 889.71 & 3.98 & 5.95 & 4.965 & 6.796 & 24.8 \\
$\mathbf{5}^{\text {th }} \mathbf{T S}$ & $22-24$ & 890.32 & 3.98 & 5.93 & 4.955 & 6.827 & 24.82 \\
$\mathbf{6}^{\text {th }} \mathbf{T S}$ & $6-28$ & 890.82 & 3.95 & 5.62 & 4.785 & 6.847 & 24.67 \\
$\mathbf{7}^{\text {th }} \mathbf{T S}$ & $6-10$ & 890.93 & 3.95 & 6.44 & 5.195 & 6.838 & 24.87 \\
$\mathbf{8}^{\text {th }} \mathbf{T S}$ & $4-12$ & 891.66 & 3.93 & 6.77 & 5.35 & 6.813 & 24.62 \\
\hline
\end{tabular}

the line. But the line (24 - 25) is the optimal line to be switched among the candidate lines; by switching this line, the losses and cost are almost reduced.

\section{Impacts of Switching on System Parameter}

The transmission line switching affects the system parameter such as LMP, generation dispatch and voltage pro- 
file which are discussed as follows:

\section{1) LMP Variation:}

LMP is the cost of supplying the next MW of load at a specified location and includes the effect of transmission congestion and losses. Table 3 shows that the LMP variation of 6-bus system during normal condition and after switching, the average value of LMP decreases from 0.2 to $7.7 \%$ due to switching [16]. Among these 2 switching the $1^{\text {st }}$ TS has large variation LMP. The LMP variation is from -0.46 to $14.65 \%$ in IEEE-30 bus system due to switching. The decrease in the LMP value also reduces the congestion rent. This variation is shown in Table 2.

\section{2) Generation Dispatch Variation:}

The generation dispatch variation in 6-bus system is shown in Table 4, before and after switching. Similarly, the IEEE-30 bus system has 8-candidate lines to be switched, to reduce the losses of system; here also the generation dispatch varies at each switching, but the dispatch variation due to switching of line (25 - 27) is only shown in Table 5.

\section{3) Voltage Profile:}

The voltage at the each load bus varies from minimum to maximum voltages [17], when the switching actions are allowed. In 6-bus system the voltage at each bus does not change due to switching of line (1 - 2) and (2 - 3) as shown in Figure 6. Whereas the opening of line (25 - 27) in IEEE-30 bus system, the results shows that the voltage increase for most of the buses and it remain same for few buses as shown in Figure 7. However the average of bus voltage increase is from 1.0 to 1.05 p.u, also in the pre-switching system the minimum voltage is 0.95 p.u at bus 5 ; after switching of line the minimum voltage raised to 0.975 at bus 5 and it is noted that the voltage at all buses are above 0.97 ensuring voltage security.

\section{4) Tighter Voltage Band:}

The minimum and maximum voltage band that has to be maintained by each bus in power system is $0.95 \leq \mathrm{V}$ $\leq 1.06$. Table 6 shows the tighter voltage band on IEEE-30 bus system before and after switching of line which

Table 3. LMP variation in 6-bus system.

\begin{tabular}{cccc}
\hline \multirow{2}{*}{ System state } & & LMP(\$MWh) & Average \\
\cline { 2 - 4 } & Min & Max & 13.615 \\
Base case & 11.56 (Bus2) & 15.67 (Bus4) & 12.565 \\
$\mathbf{1}^{\text {st }} \mathbf{T S}$ & 11.77 (Bus2) & 13.36 (Bus4) & 13.575 \\
$\mathbf{2}^{\text {nd }} \mathbf{T S}$ & 11.50 (Bus2) & 15.65 (Bus4) \\
\hline
\end{tabular}

Table 4. Generation dispatch.

\begin{tabular}{cccc}
\hline System variables & Base case & $\mathbf{( 1 - 2 ) ~ T S}$ & $(\mathbf{2}-\mathbf{3}) \mathbf{T S}$ \\
\hline $\mathbf{P G}_{\mathbf{1}}(\mathbf{M W})$ & 77.22 & 61.05 & 75.28 \\
$\mathbf{P G}$ (MW) & 69.27 & 81.04 & 65.76 \\
$\mathbf{P G}_{3}$ (MW) & 70.42 & 74.53 & 75.78 \\
\hline
\end{tabular}

Table 5. Generation dispatch.

\begin{tabular}{rcc}
\hline System variables & Pre-switching & Post-switching (25 - 27) \\
PG1 (MW) & 80 & 80 \\
PG2 (MW) & 79.88 \\
PG13 (MW) & 28.83 & 27.79 \\
PG22 (MW) & 41.12 & 47.66 \\
PG23 (MW) & 26.63 & 24.72 \\
PG27 (MW) & 33.83 & 32.41 \\
\hline
\end{tabular}




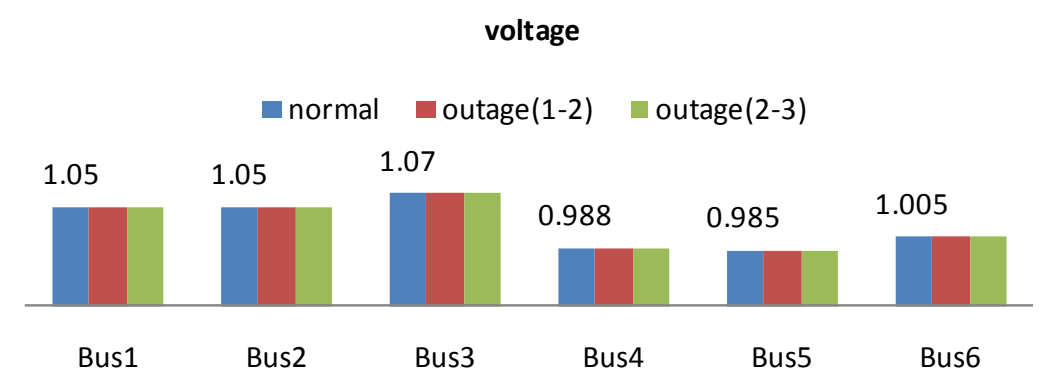

Figure 6. Voltage variation for 6-bus system.

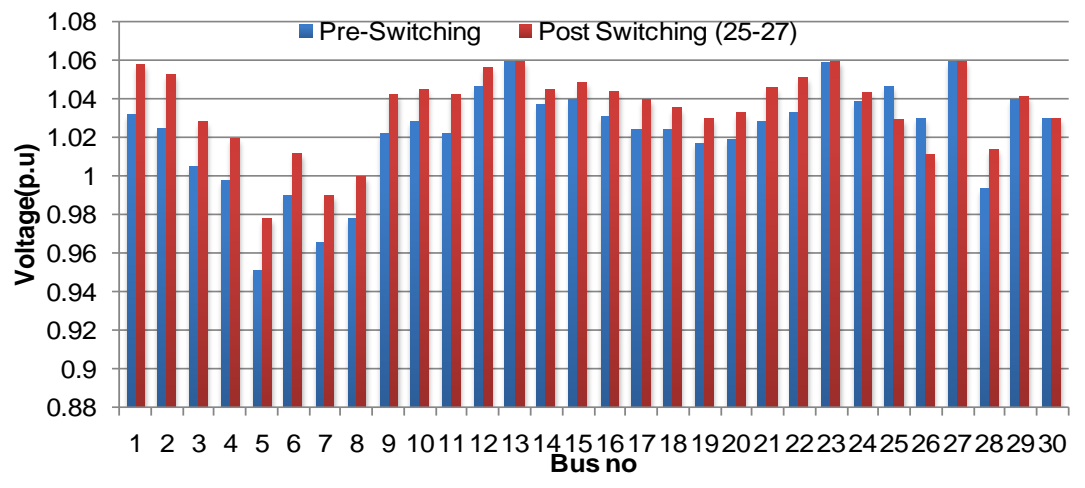

Figure 7. Voltage variation.

Table 6. Voltage limits (min and max).

\begin{tabular}{ccc}
\hline System state & Minimum voltage (p. u) & Maximum voltage (p. u) \\
\hline Base case & 0.951 (Bus 5) & 1.06 (Bus 27) \\
$\mathbf{1}^{\text {st }} \mathbf{T S}$ & 0.978 (Bus 5) & 1.06 (Bus 27) \\
$\mathbf{2}^{\text {nd }} \mathbf{T S}$ & 0.97 (Bus 5) & 1.06 (Bus 27) \\
$\mathbf{3}^{\text {rd }} \mathbf{T S}$ & 0.952 (Bus 5) & 1.06 (Bus 27) \\
$\mathbf{4}^{\text {th }} \mathbf{T S}$ & 0.952 (Bus 5) & 1.06 (Bus 27) \\
$\mathbf{5}^{\text {th }} \mathbf{T S}$ & 0.952 (Bus 5) & 1.06 (Bus 22) \\
$\mathbf{6}^{\text {th }} \mathbf{T S}$ & 0.979 (Bus 5) & 1.06 (Bus 1) \\
$\mathbf{7}^{\text {th }} \mathbf{T S}$ & 0.951 (Bus 5) & 1.06 (Bus 27) \\
$\mathbf{8}^{\text {th }} \mathbf{T S}$ & 0.953 (Bus 5) & 1.06 (Bus 27) \\
\hline
\end{tabular}

ensure the system security limit are not violated after switching of line.

\section{Determination of Priority List}

By using the TS based on the proposed method, one can find the candidate lines that need to be opened based on reduction of generation cost as shown in Table 2. The system operator can make use of this priority list to open a line to reduce the system losses, but in practice only one line is allowed to open in order to maintain $N-1$ contingency. As a result of switching the transmission line based on priority list made the system losses are reduced and voltage profile is improved as shown in Table 6.

\section{Conclusions}

In this paper, total cost and loss reduction using transmission switching (TS) considering $N-1$ contingency and 
voltage security criteria is presented and discussed. The TS is formulated as a tool for reduction of losses, cost and congestion relief in the system by reducing the locational marginal pricing (LMP) and the problem is solved using ACOPF by Interior Point Method. In some cases more than one TS may be chosen. The effect of transmission switching on power generation, voltage change, generation cost and congestion relief are also discussed. Any switching action which would violate voltage security and/or $N-1$ security criteria is not considered as candidate line. The priority list is made by considering total generation cost and the TS with AC constraints are also discussed. The priority list can also be made by considering LMP inorder to relief the congestion in the system and to reduce the system losses and improve the voltage.

The main contribution of this paper is summarized below:

- Interior Point Method using Primal/Dual Method is employed effectively to solve the problem.

- A priority list is made for switching the lines.

\section{References}

[1] Wrubel, J., Rapiciennski, P., Lee, K. and Gisin, B. (1996) Practical Experience with Corrective Switching Algorithm for On-Line Application. IEEE Transactions on Power Systems, 11, 415-421. http://dx.doi.org/10.1109/59.486127

[2] Koglin, H. and Muller, H. (1982) Corrective Switching: A New Dimension in Optimal Load Flow. International Journal of Electrical Power \& Energy Systems, 4, 142-149. http://dx.doi.org/10.1016/0142-0615(82)90041-2

[3] Khanabadi, M., Ghasemi, H. and Doostizadeh, M. (2013) Optimal Transmission Switching Considering Voltage Security and $N-1$ Contingency Analysis. IEEE Transactions on Power Systems, 28, 542-550. http://dx.doi.org/10.1109/TPWRS.2012.2207464

[4] Mazi, A., Wollenberg, B. and Hesse, M. (1986) Corrective Control of Power System Flows by Line and Bus-Bar Switching. IEEE Transactions on Power Systems, 1, 258-265. http://dx.doi.org/10.1109/TPWRS.1986.4334990

[5] Capitanescu, F., Glavic, M., Ernst, D. and Wehenkel, L. (2006) Interior-Point Based Algorithms for the Solution of Optimal Power Flow Problems. Electric Power Systems Research, 77, 508-517.

[6] Shahidehpour, M. and Ramesh, V. Nonlinear Programming Algorithms and Decomposition Strategies for OPF. IEEE/ PES Tutorial on Optimal Power Flow.

[7] Sousa, A.A. and Torres, G.L. (2010) Robust Optimal Power Flow Solution Using Trust Region and Interior-Point Methods. IEEE Transactions on Power Systems, 26, 487-499.

[8] Capitanescu, F., Glavic, M. and Wehenkel, L. An Interior Point Method Based Optimal Power Flow.

[9] William Rosehart, Jose A. Agudo Alternat Jr. (2006) On Efficient Implementation of Interior-Point Based Optimal Power Flows in Rectangular Coordinates. IEEE Transactions on Power Systems, 1747-1752.

[10] Xia, Y., Chan, K.W. and Liu, M. (2005) Direct Nonlinear Primal-Dual Interior-Point Method for Transient Stability Constrained Optimal Power Flow. IEEE Proc.-Gener. Transm. Distrib., 152.

[11] Fu, Y., Shahidehpour, M. and Li, Z. (2006) AC Contingency Dispatch Based on Security-Constrained Unit Commitment. IEEE Transactions on Power Systems, 21, 897-908. http://dx.doi.org/10.1109/TPWRS.2006.873407

[12] Wood, A.J. and Wollenberg, B.F. (2003) Power Generation, Operation and Control.

[13] Fisher, E.B., O’Neill, R.P. and Ferris, M.C. (2008) Optimal Transmission Switching. IEEE Transactions on Power Systems, 23, 1346-1355. http://dx.doi.org/10.1109/TPWRS.2008.922256

[14] http://www.ee.washington.edu/research/pstca/

[15] Bacher, R. and Glavitsch, H. (1988) Loss Reduction by Network Switching. IEEE Transactions on Power Systems, 3, 447-454. http://dx.doi.org/10.1109/59.192895

[16] Hedman, K., O’Neill, R., Fisher, E. and Oren, S. (2009) Optimal Transmission Switching with Contingency Analysis. IEEE Transactions on Power Systems, 23, 1577-1586. http://dx.doi.org/10.1109/TPWRS.2009.2020530

[17] Bacherand, R. and Glavitsch, H. (1986) Network Topology Optimization with Security Constraints. IEEE Transactions on Power Systems, 1, 103-111. http://dx.doi.org/10.1109/TPWRS.1986.4335024 


\section{NOMENCLATURE}

Indices:

$i, i \rightarrow$ index of bus

Variables:

$n \rightarrow$ Number of buses

$g \rightarrow$ number of generators

$V \rightarrow$ voltage

$G \rightarrow$ Branch conductance

$B \rightarrow$ Branch susceptance

$P_{g i} \rightarrow$ real power generation @ $i^{\text {th }}$ bus

$Q_{g i} \rightarrow$ reactive power generation @ $i^{t h}$ bus

$P_{d i} \rightarrow$ real power demand @ $i^{\text {th }}$ bus

$Q_{d i} \rightarrow$ reactive power demand @ $i^{\text {th }}$ bus

$e \rightarrow$ real part of voltage

$f \rightarrow$ imaginary part of voltage

$S \rightarrow$ shunt elements

$d \rightarrow$ load $\vee$ demand

$l \rightarrow$ transmission lines

$t \rightarrow$ transformer

$T C \rightarrow$ Total generation cost $\in \$ \mathrm{~h}$

$a \rightarrow$ transformer ratio 